\title{
Becoming an Editor
}

Hello and welcome! This course is designed to help you become a successful editor of a scholarly journal. Whether you are starting your own journal or taking on the responsibility for an existing journal, you'll find all of the basics covered in this course, as well as pointers toward more advanced topics.

If you work through all of the lessons in this course, you will be able to:

1. Perform the major tasks required by an Editor for a scholarly journal

2. Analyze and solve common problems that may arise when editing a scholarly journal

3. Assist others members of the journal team

4. Know where to look for help when you don't know what to do

See the modules here: http://pkpschool.sfu.ca/becoming-an-editor/ 